\title{
Differentiating radiation changes from local recurrence after SBRT for lung cancer: the need for better decision guidelines
}

\begin{abstract}
Radiographic lung changes and fibrosis are near ubiquitous observations on CT imaging following the treatment of non-small cell lung cancer with stereotactic body radiation therapy (SBRT). The distinction between these radiographic changes and a local cancer recurrence in a timely and accurate fashion is critical for successful salvage therapy as well as to avoid potentially detrimental overtreatment. Nonetheless, an adequate, widely accepted and validated approach for post-treatment surveillance and identification of recurrences is unavailable. This article relates a case wherein the failure of close follow up imaging and uncertainty in the interpretation of imaging resulted in overtreatment. It also identifies some of the shortcomings in the current attempts to develop an appropriate post-SBRT follow up schedule, examines the physiological and radiographic post-SBRT radiation changes and reviews the pertinent literature.
\end{abstract}

Keywords: non-small cell lung cancer, stereotactic body radiotherapy, recurrence, salvage therapy
Volume 4 Issue 4 - 2017

\author{
Bernard Newman,' Rachit Shah, ${ }^{2}$ Elisabeth \\ Weiss' \\ 'Department of Radiation Oncology, Virginia Commonwealth \\ University, USA \\ ${ }^{2}$ Department of Cardiothoracic Surgery, Virginia \\ Commonwealth University, USA
}

Correspondence: Elisabeth Weiss, Department of Radiation Oncology, Virginia Commonwealth University, 40 I College Street, Richmond,VA 23298, USA

Email elisabeth.weiss@vcuhealth.org

Received: October 29, 2017 | Published: November 10, 2017
Abbreviations: CT, computed tomography; DLCO, diffusion capacity for carbon monoxide 4D four dimensional; ESMO, european society for medical oncology; FEV1, forced expiratory volume in the first second; IMRT, intensity modulated radiotherapy; MV, mega electron Volt; NCCN, national comprehensive cancer network; NSCLC, nonsmall cell lung cancer; PET, positron emission tomography; PFTs, pulmonary function tests; SBRT, stereotactic body radiation therapy; SUV, standard uptake value in PET

\section{Introduction}

Stereotactic body radiation therapy (SBRT) has rapidly been adopted as a treatment option for early stage nonsmall cell lung cancer (NSCLC), particularly for medically inoperable patients. ${ }^{1}$ Most patients treated for NSCLC with SBRT develop posttreatment radiographic changes which often make it difficult to distinguish between lung fibrosis and cancer recurrence. ${ }^{2-6}$ The need for protocols that facilitate a correct diagnosis has been the subject of several investigations. ${ }^{2,3,7,8}$ Our case report demonstrates these diagnostic challenges and the need for an appropriate follow up protocol.

\section{Case presentation}

A 61yearold Caucasian male recent smoker with severe emphysema, but good functionality, presented for evaluation of a recently discovered left lung lesion. Pertinent history included a 40 packyear smoking history, right lung wedge resection for an abnormal lung lesion on chest x-ray 6years earlier (pathology showed scar tissue) and a family history positive for lung cancer. On exam, Karnofsky index was $90 \%$ and PFTs revealed a FEV1 of 1.71 and a DLCO of $60 \%$ predicted. Resting O2 saturation was $94 \%$ and the physical exam was unremarkable. Radiological studies included a chest CT scan showing a $1.2 \mathrm{~cm}$ spiculated lesion and a PET-CT scan showing a lesion with SUVmax of 3.2, with no other areas of increased SUV uptake.
Initial assessment was that the risk of pneumothorax would be significant for biopsy procedures due to emphysematous tissue surrounding the lesion, and he was referred to Radiation Oncology to be evaluated for SBRT in the absence of a tissue diagnosis. Surgery was considered an option, but not without the high-risk tissue biopsy to prove malignancy, and though he had recently stopped smoking he was considered high-risk for relapse making him a less desirable surgical candidate. Furthermore it was felt that SBRT was less likely to be detrimental to his pulmonary function than a surgical resection and the patient preferred a less invasive option.

Upon evaluation Radiation Oncology agreed SBRT without biopsy was a valid treatment alternative to surgery with a preceding biopsy. ${ }^{9}$ Repeat CT and PET scans showed the lesion was expanding and demonstrated increasing PET positivity with a maximum SUV of 4.4 making malignancy very likely, and per NCCN guidelines should be resected without biopsy due to it's baseline size and interval enlargement. This approach lacked a tissue confirmation of malignancy but the positive and increasing metabolic activity on PET scan, which are used to aid in the diagnosis of moderate risk lesions in the NCCN algorithm, did provide additional confidence in the diagnosis and course of action. The lesion, assumed to be a stage IA non-small cell lung cancer, was treated with SBRT to 48Gy in four fractions every otherday using 10 static IMRT fields with $6 \mathrm{MV}$ photons based on 4D CT for treatment planning. The patient tolerated the procedure well and was scheduled for routine three monthly follow up visits with imaging.

One month post SBRT the patient had no clinical or radiographic signs of radiation pneumonitis. A chest $\mathrm{CT}$ at 3 months revealed no evidence of recurrent or progressive disease and his concurrent visit was unremarkable. His 7 months post-treatment $\mathrm{CT}$ revealed a slight decrease in lesion size with spiculation and dendritic extensions into the adjacent lung, extending to the pleura, with new soft tissue architectural distortions, most consistent with radiation changes. 
The patient failed to return at 12 months post-treatment for follow up, and did not have his scan or visit until nearly 16 months post-SBRT. This chest CT scan revealed an interval enlargement of the left upper lobe spiculated mass to a size of $2.7 \times 5.5 \mathrm{~cm}$, with associated reorientation of the surrounding bronchovascular structures and dendritic extensions to the pleural surfaces and major fissure. Accompanying left upper lobe volume loss was also observed. Noteworthy high-risk features included craniocaudal growth and bulging margins. Figure 1 for evolving lesion imaging and treatment planning. A PET scan showed an increased SUVmax of 8.5 in the left upper lobe without additional metastatic disease. The patient underwent a CT-guided lung biopsy resulting in 8 samples being obtained prior to a complicating pneumothorax requiring hospitalization. Pathology was negative for cancer.
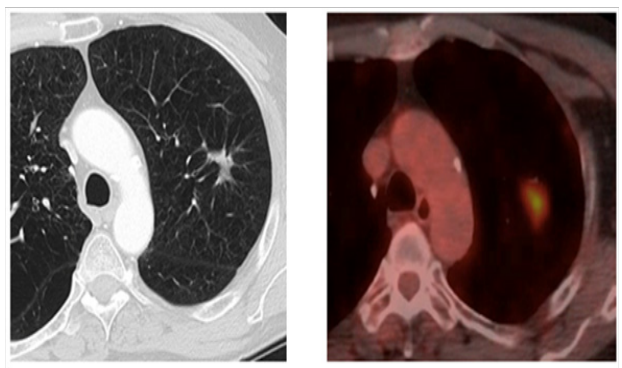

Figure A Pre-treatment CT and PET-CT.
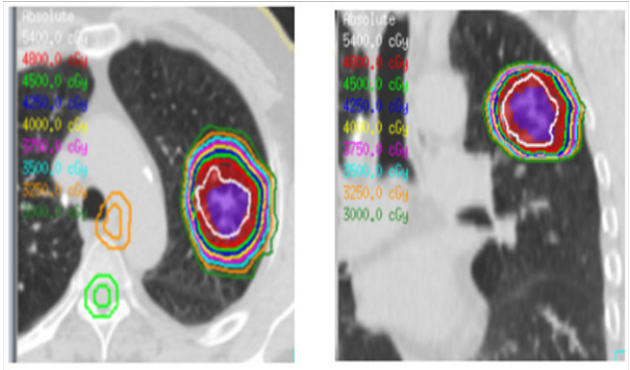

Figure B SBRT treatment plan.
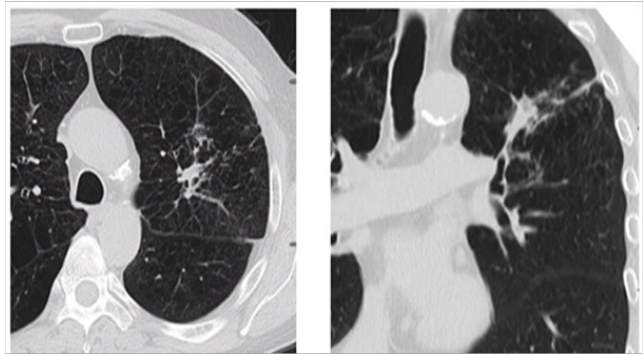

Figure C 7month post-SBRT CT scan.
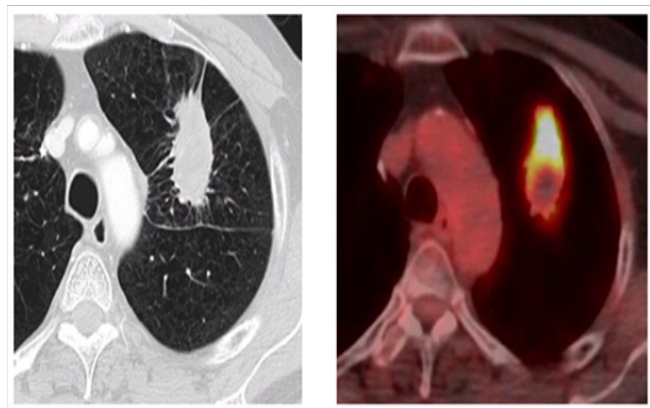

Figure D 16month axial CT and PET-CT scans concerning for local recurrence.

Figure I Presentation, treatment and evolution of left upper lobe lesion.
With new CT imaging 17months post-SBRT the Tumor Board felt that the lesion likely was a local recurrence based on imaging. Two weeks later the patient therefore underwent a left thoracoscopy converted to a left lateral thoracotomy, left upper lobectomy and mediastinal lymph node dissection. The surgical pathology report noted that extensive parenchymal necrosis and multiple nonnecrotizing granulomas with no viable tumor were identified in the specimen submitted. The patient developed an air leak after surgery and was not discharged until 9days post-op.

\section{Discussion}

Differentiating between postSBRT lung changes and local recurrences is challenging and the subject of ongoing research..$^{2-6,10-12}$ Our patient's case highlights many of these uncertainties and difficulties. Due to the fear of causing morbidity there was no preSBRT tissue diagnosis. PostSBRT attempts at a tissue diagnosis did in fact cause significant harm and the biopsy results obtained were ultimately disregarded as false negatives in the face of imaging that was very concerning for local recurrence. To aid with the distinction between post-radiation pulmonary changes and local cancer recurrence, the sequential radiographic and physiologic changes and physical findings found after SBRT treatment should be considered.

The physiological response to radiation occurs in 3 sequential phases. First there is an acute exudative phase, then an organizing or proliferative phase and lastly a chronic fibrotic phase. The first 2 phases are characterized by a macrophage infiltrate, and are often grouped together as the acute phase. During the acute phase most patients are asymptomatic or subclinical, but clinical findings do occur in around $928 \%$ of patients. Radiation pneumonitis corresponds to the acute phase and typically occurs during the first 6 months post-SBRT. Pulmonary fibrosis corresponds to the subsequent chronic phase and typically occurs 6 to 12 months post-SBRT, and may continue up to 24months. ${ }^{12-16}$

The radiological changes induced by pulmonary SBRT are specific and differ in terms of topography and chronology from those of other radiation therapy modalities. Radiation pneumonitis may appear as ground-glass opacities, consolidation or both. These early post-SBRT findings may also be described as diffuse consolidation, diffuse ground glass opacity, patchy heterogeneous consolidation with ground glass opacity and a mosaic of ground glass opacity. Ground glass appearance is common, and sub-categories are recognizable to radiation oncologists and others familiar with $\mathrm{CT}$ interpretation. ${ }^{17,18}$ The late phase of radiation fibrosis frequently appears as a well-defined area of volume loss with a linear scar or consolidation, parenchymal distortion and traction bronchiectasis that conforms to the treatment portals which may either stabilize or evolve up to 24 months. Shrinkage of the region of fibrotic consolidation or a more sharply defined demarcation between normal and irradiated lung parenchyma may occur as the process progresses. These findings are sometimes associated with ipsilateral displacement of the mediastinum and adjacent pleural thickening or effusion..$^{17,18}$

The use of PET scans to aid in the early identification and differentiation between radiation fibrosis and recurrence, has been discussed in the literature. ${ }^{2,3,5}$ While some sources caution against a high reliance on PET scans as being useful as discriminators between post-SBRT recurrence and fibrosis, others have postulated a SUV of greater than 5 can be useful to that determination. Our case clearly supports a cautionary approach. Actually, in the case at hand, a high SUV of 8.5 supported the Tumor Board assumption of a tumor recurrence. At this time, neither the NCCN nor ESMO guidelines 
recommend routine post-SBRT PET scans at a specified interval as part of regular post-treatment surveillance. Incorporation of PETCT into post-SBRT cancer surveillance is currently the subject of research. ${ }^{7,18}$

Knowledge of the radiation techniques and portals used can help analyze post-SBRT radiographic changes. On CT the sequential enlargement of the suspected lesion, bulging margins, linear margin disappearance, cranio-caudal growth and the loss of an air bronchogram are some high risk features of a lesion that can be useful in differentiating between radiation pneumonitis/ fibrosis and recurrence. ${ }^{10,11}$ Nodules outside the zone of radiation fibrosis, bone destruction, pleural effusion long after treatment and mediastinal involvement all could lead towards the diagnosis of local recurrence. . $^{2,3,14}$

Several factors made the differentiation and treatment decisions particularly difficult in our case. A marked increase in lesion size and increase in SUVmax heavily influenced the decision making process. Perhaps even more significantly the patient missed a follow up visit and went 8 months without post-treatment surveillance. This gap denied the opportunity to sooner detect and closely observe the evolving lesion.

Currently, no ideal post-SBRT follow up schedule has been developed. Due to the high local control rates with SBRT, studies with large patient numbers that differentiate between local recurrence and non-malignant lung changes are difficult to perform. In a study by Verstegen et al. ${ }^{8}$ local recurrences were diagnosed via convincing CT imaging with or without the aid of a variety of modalities including PET scans, follow up CT imaging and, to a lesser extent, pathology. When local recurrences could not be differentiated from post-radiation fibrosis they were counted as a local recurrence. Based on this study's criteria, our patient would have incorrectly been counted as having a local recurrence. The effect of this error in an outcome-oriented study would likely have been significant given the few patients actually considered to have a recurrence. The European Society for Medical Oncology's post-SBRT follow up schedule had initially recommended CT imaging following SBRT "every 3 to 6months for 2 to 3years" and recently changed that recommendation to "every 6months for at least 3years". ${ }^{7}$ The Verstegen study endorsed the new surveillance schedule, but did not demonstrate the safety or efficacy of the increased interval between CT scans. NCCN guidelines acknowledge the need for close surveillance post-SBRT with a team of experts familiar with SBRT due to difficulty differentiating post-radiation changes from recurrence. ${ }^{19}$ At this time a specifically tailored post-SBRT surveillance schedule is not given by the NCCN and a single surveillance schedule which has been used for other modalities of treatment (i.e. surgery and conventional chemo-radiation) of CT every 6-12months for 2years and low dose non contrast CT annually thereafter is given, with the caveat that patients treated with chemotherapy, radiation or have residual abnormalities may require more frequent imagining of unspecified intervals. Based on our case, there is concern that longer follow up intervals, which would eclipse the entire exudative phase, can lead to situations similar ours where the early development of a lesion was not monitored and therefore a local recurrence could not be differentiated from post-radiation changes. In addition, early detection of recurrences is crucial for patients who are candidates for salvage therapy while the disease is still locally confined. Our goal should be to capture the steps in the radiographic evolution process as best we can in each patient so we can more easily differentiate this process from recurrence. The best way to do this may be with close, even patient specific, follow up once the beginning of change is observed. To this end current research is investigating the role of high risk radiographic features to differentiate between local recurrence and post-radiation changes. Mattonen et al. ${ }^{10}$ examined the role of radiomic assessment, and is particularly pertinent, because they found that with the aid of computers local recurrences may be detected earlier than by physician eyes alone which could have a significant impact for patients in cases similar to ours. Peulen et al. ${ }^{11}$ examined high risk CT features and developed a simplified model that found the combination of cranio-caudal growth and bulging margins to be a good indicator for recurrence. ${ }^{11}$

\section{Conclusion}

Our case serves as a cautionary tale. It shows the profound difficulty of identifying local recurrences after SBRT. A closer, more cautious follow up protocol with a chest CT every 34 months to better differentiate between post-radiation changes and local recurrence might be beneficial. Parameters that reliably characterize recurrences need to be identified.

\section{Acknowledgements}

None.

\section{Conflict of interest}

Author declares that there is no conflict of interest.

\section{References}

1. Yu XJ, Dai WR, Xu J. Survival Outcome after Stereotactic Body Radiation Therapy and Surgery for Early Stage Non-Small Cell Lung Cancer: A Meta-Analysis. J Invest Surg. 2017;22:1-8.

2. Huang K, Dahele M, Senan S, et al. Radiographic changes after lung stereotactic ablative radiotherapy (SABR) - Can we distinguish recurrence from fibrosis? A systematic review of the literature. Radiother Oncol. 2012;102(3):335-342.

3. Huang K, Senthi S, Palma DA, et al. High-risk CT features for detection of local recurrence after stereotactic ablative radiotherapy for lung cancer. Radiother Oncol. 2013;109(1):51-57.

4. Hassaballa HA, Cohen ES, Khan AJ, et al. Positron emission tomography demonstrates radiation-induced changes to nonirradiated lungs in lung cancer patients treated with radiation and chemotherapy. Chest. 2005;128(3):1448-1452.

5. Chen F, Matsuo Y, Yoshizawa A, et al. Salvage lung resection for nonsmall cell lung cancer after stereotactic body radiotherapy in initially operable patients. J Thor Oncol. 2010;5(12):1999-2002.

6. Bibault JE, Ceugnart L, Prevost B, et al. CT appearance of pulmonary carcinomas after stereotactic radiation therapy. Diagn Interv Imaging. 2013;94(3):255-262.

7. Vansteenkiste J, Crinò L, Dooms C, et al. 2nd ESMO Consensus Conference on Lung Cancer: early-stage non-small-cell lung cancer consensus on diagnosis, treatment and follow-up. Ann Oncol. 2014;25(8):1462-1474.

8. Verstegen NE, Lagerwaard FJ, Hashemi SM, et al. Patterns of disease recurrence after SABR for early stage non-small cell lung cancer: Optimizing follow-up schedules for salvage therapy. J Thorac Oncol. 2015;10(8):1195-1200. 
9. Singh DP, Bergsma DP, Cummings MA, et al. Outcome with Stereotactic Body Radiation Therapy for Stage I Non Small Cell Lung Cancer Using 5 Fractions: Single Institution Experience of 106 Consecutive Patients. Int J Radiat Oncol Biol Phys. 2017;98(1):231-232.

10. Mattonen SA, Palma DA, Johnson C, et al. Detection of local cancer recurrence after stereotactic ablative radiation therapy for lung cancer: Physician performance versus radiomic assessment. Int J Radiat Oncol Biol Phys. 2016;94(5):1121-1128.

11. Peulen H, Mantel F, Guckenberger M, et al. Validation of high-risk computed tomography feature for detection of local recurrence after stereotactic body radiation therapy for early-stage non-small cell lung cancer. Int J Radiat Oncol Biol Phys. 2016;96(1):134-141.

12. Choi YW, Munden RF, Erasmus JJ, et al. Effects of radiation therapy on the lung: Radiologic appearances and differential diagnosis. Radiographics. 2004;24(4):985-998.

13. Provatopoulou X, Athanasiou E, Gounaris A. Predictive markers of radiation pneumonitis. Anticancer Res. 2008;28(4):2421-2432.
14. Aoki T, Nagata Y, Negoro Y, et al. Evaluation of lung injury after three-dimensional conformal stereotactic radiation therapy for solitary lung tumors: CT appearance. Radiology. 2004;230(1):101-108.

15. Larici AR, del Ciello A, Maggi F, et al. Lung abnormalities at multimodality imaging after radiation therapy for non-small cell lung cancer. Radiographics. 2011;31(3):771-789.

16. Kalman N, Hugo GD, Kahn JM, et al. Inter-observer Reliability in Describing Radiographic Lung Changes after Stereotactic Body Radiotherapy. Int J Radiat Oncol Biol Phys. 2017;99(25):S196.

17. Trovo M, Linda A, El Naqa I, et al. Early and late lung radiographic injury following stereotactic body radiation therapy (SBRT). Lung Cancer. 2010;69(1):77-85.

18. Linda A, Trovo M, Bradley JD. Radiation injury of the lung after stereotactic body radiation therapy (SBRT) for lung cancer: A timeline and pattern of CT changes. Eur J Radiol. 2011;79(1):147-154.

19. https://www.ncen.org/professionals/physician_gls/pdf/nscl.pdf 